\title{
Sociedad de la información: Los mecanisnos reguladores en el contexto de una sociedad emergente
}

\author{
Yohannis Marti \\ Licenciada en información científico-técnica y bibliotecología. \\ Maestrante en bibliotecología y ciencia de la información. Profesora \\ del Dpto. de Bibliotecología y Ciencia de la Información. \\ E-mail: yohannis@fcom.uh.cu \\ Rosa Lidia Vega-Almeida \\ Licenciada en información científico-técnica y bibliotecología. \\ Maestrante en bibliotecología y ciencia de la información. Profesora \\ del Dpto. de Bibliotecología y Ciencia de la Información. \\ E-mail: vega.rosa@infomed.sld.cu
}

\section{INTRODUCCIÓN}

El desarrollo alcanzado por la especie humana no sólo ha generado beneficios, ha suscitado también problemas y antagonismos que sumergen al mundo de hoy en una profunda crisis que afecta todo y a todos.

En este contexto, el término sostenibilidad-ampliamente difundido desde las postrimerías del pasado siglo ante la cierta inminencia del advenimiento del III Mileniose ha convertido en palabra clave del discurso político; evidenciando la preocupación explícita pero no unánime por el futuro de esta "aldea global". Futuro ¿imprevisible? que encierra, en sí mismo, la preocupación por la preservación de nuestra propia especie en peligro de extinción.

El ser humano -"resumen viviente del decurso histórico de la humanidad"(GONZÁLEZ, 2000)-, ser social que asimila, asume, aprehende y se apropia de la experiencia acumulada en la interacción con sus semejantes y el mundo, indudablemente es el único reponsable de sus aciertos y desaciertos; y tiene ante sí, el reto de mobilizar todos los recursos necesarios para la consecución de la perdurabilidad común.

Sociedad de la información; Ética de la información: Política de información; Legislación de la información; Mecanismos de regulación social.

\section{Information society: regulator mechanims in an emergent societal context}

\begin{abstract}
The information society like new context of human relations is examined. The ethics, the policy and the legislation as their regulating mechanisms at social and individual level are analyzed. The new ethical, political and legislative considerations relative to the information are exposed. Final considerations are offered that turn on the importance of the ethical and moral education, the construction and consolidation of the informational culture and the information literacy like processes oriented to the improvement and progress of the individuals and the society.
\end{abstract}

\section{Key words}

Information society; Information Ethics; Information policy; Legislation of Information; Regulator mechanisms of social behaviour.
El desarrollo social logrado innegablemente, también ha insertado cambios asociados a un emergente y flamante recurso. Esas transformaciones revolucionan la concepción de la información -"factor central y estratégico para el progreso social y económico,"(UNITED NATIONS, 2000) y los procesos de informar e informarse. Fenómenos que afectan a la sociedad en su conjunto desde la propia noción y filosofía del mundo hasta las formas y categorías de organización a escala social, lo cual ha provocado la emergencia de la denominada Sociedad de la Información.

Sociedad cuya existencia no depende exclusivamente del desarrollo material determinante en la evolución hacia nuevos tipos de sociedades; pues, su proyección exige que afloren de esas "multiplicidades interiores" (MORIN, 1999) del ser humano nuevas ideas, creencias y valores preeminentes y universales.

La humanidad transita hacia una nueva sociedad que al mismo tiempo construye, pues de otra manera no 
puede ser. En este decursar, experimenta e implementa transformaciones que sincrónica e inevitablemente imponen la formación de un nuevo ser social.

Ser social que inmerso en el proceso de transición de la Sociedad Industrial a la Sociedad de la Información debe responder a nuevos imperativos éticos, políticos y legislativos relativos a la información.

Precisamente, las siguientes páginas están dedicadas a discurrir sobre los mencionados imperativos. Sin embargo, el abordaje del tema en cuestión precisa partir de la caracterización del escenario proyectado y manifestado.

\section{SOCIEDAD DE LA INFORMACIÓN: UN NUEVO CONTEXTO}

Muchos son los que esgrimen argumentos para fundamentar la necesidad de crear una nueva sociedad. Sociedad nombrada de la información por unos, y del conocimiento o del aprendizaje por otros. En ella información y conocimiento son recursos críticos que impactan la economía y la vida social.

Por consiguiente, los recursos clave de la sociedad industrial son sustituidos por recursos estratégicos revalorizados y de alta significación económica, política y social. De esta manera, la información y el conocimiento pautan el tránsito de la tangibilidad a la intangibilidad de los bienes; es decir, de "la industria productora de objetos a la producción de servicios y una economía basada en el conocimiento,"(MOREIRO, 1998) potencializada por la utilización intensiva y extensiva de las Tecnologías de la Información y la Comunicación.

Sin lugar a dudas, tal circunstancialidad histórica supone necesariamente implantar mecanismos reguladores a nivel individual y social en el contexto de esta sociedad emergente. Mecanismos que han de estar focalizados a la información y el conocimiento como perdurables elementos medulares del progreso humano.

Es válido destacar que mientras la sociedad intenta establecer condiciones y condicionantes que un tanto normalizan y regulan los procesos de percepción, análisis, tratamiento y difusión inherentes al fenómeno información, el propio fenómeno siempre ha actuado como elemento determinante de la estructura de los roles desempeñados por los actores de esa sociedad.

De esta manera, los patrones de acceso a la información han funcionado como patrones de inclusión/exclusión de los procesos de interacción social. Los aspectos jerarquía, socialización e identidad a nivel societal están fuertemente influidos por las situaciones de acceso a la información.
De ahí podría afirmarse que la información ha sido y es utilizada como elemento para generar exclusión y enormes diferencias en cuanto a desarrollo económico, político y social, entre y dentro de las naciones.

La información como aspecto esencial de los fenómenos excluyentes ha sido usada en todos los tiempos. Desde la comunidad primitiva donde se conservaba en secreto determinada información y conocimiento para mantener el status y reconocimiento social, atravesando por la Edad Media donde la Iglesia mantenía un monopolio informacional, hasta hoy donde la concentración regional de sólidas infoestructuras apunta hacia una nueva denominación: Info-pobres e Info-ricos.

Si bien la información ha constituido un elemento importante para la sociedad en cada una de las formaciones económico-sociales; es en las postrimerías del siglo XX cuando comienzan a evidenciarse las primeras transformaciones estructurales de la economía y la sociedad como consecuencia del crecimiento acelerado de la producción, el procesamiento y difusión de información y conocimiento, fundamentalmente asociado a las tecnologías telemáticas.

Los actuales cambios tecnológicos conducen a la modificación de la base material de la sociedad. La globalización y la interdependencia de las economías nacionales crean nuevas formas de relación entre las naciones y nuevos problemas al magnificarse el valor económico de la información. Se tiende a la profundización de la denominada brecha digital que margina a los países y sectores sociales con débiles infoestructuras. Las oportunidades económicas, políticas, educacionales, etc. pasan inadvertidas para aquellos que no tienen, o no pueden tener, la adecuada infraestructura tecnológica e informacional que permite el acceso a la información y el conocimiento.

La naturaleza del poder cada vez se define más en términos de la distribución inadecuada de la información. La desigualdad, antes asociada con los ingresos, empieza a ser asociada con factores tecnológicos y con el control político y económico del conocimiento y la información.

Es precisamente la magnitud de las dimensiones política y económica las que han motivado el debate entre los miembros, representantes, observadores de organizaciones internacionales gubernamentales y ONGs en aras de consensuar la proyección de esta sociedad fundamentada en los principios consagrados en la Carta de las Naciones Unidas, la Declaración Universal de los Derechos Humanos y la Declaración del Milenio (ONU, 2003). 
Bajo la égida de estos principios se pretende alcanzar una sociedad caracterizada por el acceso y el uso universal de información para crear, acumular y difundir el conocimiento; pues se reconoce que el conocimiento, la información y la comunicación son fundamentales para el progreso, los esfuerzos y el bienestar de la humanidad.

Esta visión optimista que enuncia lo que en el futuro pudiera ser la Sociedad de la Información está basada en la utilización de las Tecnologías de la Información y la Comunicación y como es de suponer no es compartida por todos. La idea de que estas últimas revolucionarán aspectos completos de la vida social, cultural y económica, que harán posible "la democracia directa por primera vez en la historia de la humanidad" (TOFFLER citado por GÓNZALEZ, LÓPEZ \& LUJÁN, 1996), y que al constituirse parte especialmente importante en el crecimiento acelerado de la economía, contribuirán a la erradicación de la pobreza y la promoción del desarrollo sostenible, facilitando la beneficiosa integración hacia una economía global; suscita el desconcierto a quienes muestran preocupación por la inminente pérdida de la privacidad y de la identidad cultural, "el control exacerbado sobre el ciudadano" (Erichsen, Rezende \& Bórem, 2003) y "la potenciación del Estado autoritario" (Roszak citado por Gónzalez, López \& Luján, 1996) y la promoción y difusión de antivalores; unido a la agudización de la brecha digital que acentúa cada vez más, las diferencias entre info-ricos e info-pobres.

La preservación de la privacidad, diversidad cultural y democracia reside en la regulación interna y externa de cada una de las acciones recíprocas del individuo para con la sociedad y de la sociedad para con el individuo, en la concepción de "una unidad que asegure y favorezca la diversidad, una diversidad que se inscriba en la unidad." (Morin, 1999)

Sin embargo, no se debe esperar que tales mecanismos surjan instantánea e inconscientemente, pues tal como afirma Rubestein (1964) al referirse al regulador interno "la estructuración de una nueva sociedad, de nuevas condiciones de vida social, no da origen de forma automática ni mecánica a una nueva fisonomía moral del hombre".

\section{ETICIDAD EN EL ESCENARIO INFORMACIONAL}

La moral, mecanismo regulador interno, es producto del devenir histórico y tiene un condicionamiento clasista. Su conformación resulta de la interacción e interrelación activa y consciente de los individuos entre ellos y con el mundo, que contribuye a la formación, reconstrucción y actualización de los valores, reglas, normas y conductas sociales que aseguran la convivencia, debido a su marcado carácter orientador y regulador a nivel individual y social.

En este sentido, la moral -objeto de estudio de la Éticadeviene instrumento negentrópico de la sociedad, cohesionador de los individuos y regulador de sus conductas en la búsqueda y por la consecución de la armonía y la coherencia social.

En las sociedades occidentales y orientales, en la antigüedad y el medioevo, las cuestiones relativas a la ética -como noción de orden- ocuparon una posición preponderante, debido a la constante preocupación por los problemas de índole político y moral de los individuos -determinantes para la convivencia-.

Esta preocupación ha trascendido al resto de las sociedades en el afán por establecer los límites a cumplir para garantizar el respeto a la individualidad y a la diferencia, que implica la responsabilidad individual y social; a partir del "control interior que el individuo ejerce sobre sí mismo" (Merril, 1981), lo que permite orientar y adecuar su conducta a las relaciones sociales establecidas en la sociedad, para la consecución del orden y la unidad.

El reconocimiento de la moral como elemento imprescindible del funcionamiento orgánico de la sociedad se evidencia en la Declaración de principios de la Cumbre de la Sociedad de la Información.

En el mencionado documento se expresa que la concepción y creación de la sociedad de la información resulta imposible, sin fundamentarse en valores culturales, éticos y morales, universalmente sostenidos, como la verdad, la justicia, la solidaridad, la tolerancia, la dignidad humana, la responsabilidad compartida, la transparencia y la rendición de cuentas. Asimismo la sociedad debe edificarse sin menoscabo de los valores morales, sociales y religiosos de todas las sociedades; antendiendo a los derechos humanos que contribuyan a la constitución de un entorno en el que se respeten y alienten todos los aspectos de la dignidad del ser humano, y se protejan todas las soberanías nacionales y los intereses de toda índole de los diversos sectores sin discriminación.

En este marco, la Ética como disciplina filosófica es retomada y aplicada para regular y orientar la actitud de los individuos hacia el perfeccionamiento humano, asegurando la convivencia armónica de las diversas culturas en la Sociedad de la Información.

Por consiguiente, un nuevo campo de conocimiento, la Ética de la información, irrumpe; proponiendo una 
teoría descriptiva que explora el poder de las estructuras informacionales influyentes en las formaciones culturales. Disciplina que postula una teoría emancipadora que desarrolla la crítica moral a las actitudes y tradiciones en el campo de la información a nivel individual y colectivo; lo que incluye además aspectos normativos.

Las cuestiones éticas concernientes a la diseminación de información y los problemas relacionados con el acceso público a los servicios de información también son estudiados por esta rama del conocimiento. Así se interesa por el acceso libre e igualitario a la información para la eliminación de las diferencias entre info-ricos e info-pobres.

Según Capurro (2002), las tareas medulares de la Ética de la Información como disciplina son:

- Observación y crítica de la conducta social en el campo de la información

- Análisis de las estructuras de poder determinantes de las relaciones informativas

- Crítica de los mitos informativos

- Observación de los planteos éticos en este campo

Llegado a este momento en el análisis, se puede afirmar que cada proceso informativo tiene asociado determinadas dimensiones éticas que afectan tanto a los usuarios como a los trabajadores de la información.

Para sustentar la afirmación anterior se pueden exponer una serie de criterios asociados al ciclo de vida social de la información.

- En el proceso de generación de nueva información, el autor de ese nuevo contenido, debe respetar los derechos morales y patrimoniales de aquellos autores que han aportado elementos significativos a su mensaje.

- En cuanto al tratamiento y difusión debe igualmente tenerse en cuenta que el profesional de la información como agente cultural activo en la educación de los ciudadanos debe revisar, examinar y evaluar las fuentes para prohibir o limitar la divulgación de aquellos cuya lectura se juzgue nociva. Este proceso de censura y control se ha llevado a cabo a lo largo de la historia en todos los países tanto por entidades eclesiásticas y gubernamentales así como por organizaciones privadas e individuos. La censura y control de la información presenta una disyuntiva a estos profesionales: proveer a los usuarios todo tipo de información disponible y convertirse en tenaces defensores de los derechos de libre acceso a la información; o limitar el acceso a la información, establecer filtros y transformarse en censores informacionales alejándose de su rol de agente de cambio.

Como se puede derivar de las observaciones precedentes, la sostenibilidad de la información como recurso social se basa en la ciclicidad e iteratividad de los procesos de generación, tratamiento y difusión. En este escenario de acción se origina para las organizaciones y trabajadores de la información el siguiente dilema: ¿Cómo respetar el derecho de los demás y al mismo tiempo cumplir con la misión que ante la sociedad tienen?

Lograr el indispensable equilibrio al decidir qué censurar requiere elaborar el marco ético y la política para el desempeño organizacional. En este sentido resulta imprescindible tener en cuenta:

- Código de ética de la institución que regula el patrón de conducta dentro de unos cánones aceptables de convivencia social, en correspondencia a los principios morales universales.

- Tipología de usuario a servir versus el contenido de la información. Los criterios a manejar de acuerdo a determinado información variarán en función del tipo de usuario.

\section{PolítiCA DE INFORMACIÓN: MECANISMO \\ DE REGULACIÓN EXTERNO}

$\mathrm{Al}$ abordar los elementos que se deben tener en cuenta para actuar acorde con lo socialmente establecido se hizo referencia a la política.

La política participa en la regulación de la conducta. Esta constituye la expresión formal de la asunción consciente por parte de los individuos de la regulación moral. Dicha elaboración de orden superior al desarrollarse se instituye como mecanismo de regulación externo del comportamiento individual y social.

La sociedad en su peregrinar ha creado diversas políticas que guían el accionar social en cuestiones generadoras de conflicto. Así se pueden encontrar políticas presupuestarias, económicas, educacionales, etc. que operan a diferentes niveles (internacionales, nacionales, regionales, locales, organizacionales, comunitarios, gubernamentales, etc.)

Precisamente, una de las políticas más desarrolladas en las últimas décadas ante la inminencia de un nuevo tipo de Sociedad es la política de información. 
Tomando como referente la definición de política que ofrece la Real Academia, las políticas de información constituyen aquel conjunto de principios, directrices y estrategias que orientan el curso de acción de los programas y proyectos destinados a la generación, desarrollo, distribución, difusión y uso de los recursos, servicios y sistemas de información.

Al decir de varios autores, como Linares, Patterson y Viciedo (2000), las políticas de información no son una cuestión contemporánea para restringir o promover el flujo de información. Los sectores dominantes siempre han implementado determinados mecanismos para controlar la difusión de la información que pusiera en peligro la continuidad de su poder.

Una de las causas que pudiese argumentar el auge contemporáneo de las políticas de información es la concepción de la información como mercancía, como bien intangible que sustenta el verdadero valor de mercado de las organizaciones.

En este sentido, el control de los bienes intangibles, del capital intelectual requerirá más atención política en todos los países y organizaciones a medida que los delitos informacionales aumenten (la piratería, la falsificación, el robo y el espionaje industrial).

Es por ello que entre las funciones que debe cumplir una política de información se puede señalar:

- Proveer un marco institucional y legal para regular el intercambio formal de información

Estas políticas vinculadas a la información necesitan estar estrechamente relacionadas con todas la políticas educacionales, culturales, económica y tecnológicas. La información, como se ha expresado en reiteradas ocasiones, juega un rol determinante en todos los campos de desarrollo social. Por consiguiente, si se intenta construir una sociedad basada en la información y el conocimiento es necesario articular estrategias efectivas que engranen de forma orgánica la acción social en el mundo de la información.

La información es un fenómeno en constante evolución y como recurso ha de ser gestionado teniendo en cuenta las peculiaridades que rodean su generación, tratamiento y difusión. Si los valores que toman estas peculiaridades se transforman, entonces hay que adaptar las políticas de información a estas nuevas situaciones. Es por ello que deben revisarse las políticas sistemáticamente y por supuesto garantizar su flexibilidad, su compatibilidad con los principios morales y en correspondencia con el respeto a los derechos humanos. Las políticas deben estar dirigidas a asegurar el bienestar social y no a acentuar las diferencias sociales.

\section{LEGISLACIÓN E INFORMACIÓN: IMPERATIVO SOCIAL}

Cada país tiene una sensibilidad concreta, una acentuación especial en asuntos de moral y también de seguridad, en definitiva, cada uno tiene una personalidad. Personalidad que se expresará en la manera en que se protegen los derechos de los ciudadanos y el Estado de una probable violación.

Los derechos morales y patrimoniales que tienen las personas naturales y jurídicas sobre la información pueden ser transgredidos. La violación de estos derechos son considerados delitos, pues aunque en la actualidad el acceso a la información es un derecho que puede ejercerse libremente por cualquier persona, este derecho se encuentra restringido cuando con él puedan afectarse otros derechos como la intimidad, el patrimonio económico, la libre competencia o la seguridad de un Estado.

"Las regulaciones para salvaguardar esos derechos deben estar cuidadosamente balanceadas entre los derechos de autor y los creadores con los intereses públicos, a la vez que recompensen la creación, investigación y la innovación, y además aseguren amplio acceso al conocimiento para evitar la monopolización de la información y el conocimiento" (BIOMUNDI, 1999).

Con la impronta tecnológica en el fenómeno informacional se impone como principio que los marcos jurídicos relativos al universo de la información deberán aplicarse tanto al mundo "en línea" como al que no lo está. Si bien es importante recordar que el derecho a la información, afecta tanto al que informa como al receptor y que se encuentra protegido jurídicamente, en la praxis encuentra numerosas limitaciones.

Las transformaciones que se operan en el campo tecnológico y su consecuente impacto en los procesos de informar/ informarse requieren de un análisis permanente de la regulación jurídica sobre información. Estas disposiciones legales deben contemplar, entre otros elementos:

- el derecho a la intimidad,

- la autodeterminación informativa y

- los derechos de autor

desde una visión más objetiva y acorde con la realidad donde se articule lo real y virtual armónicamente, sin 
contradicciones ni conflictos que lesionen los derechos de unos y coaccionen la tan necesaria transmisión de conocimientos.

Los cambios legales que se realizan son insuficientes para afrontar problemas como:

- iprotección de la propiedad intelectual versus libre acceso a la información y el conocimiento que redunda en desarrollo?

- ise pueden considerar como delito las acciones que cometen los hacker?

- ¿cómo penalizarlos en caso que así fueran considerados?

- ¿cómo expresar jurídicamente qué diferencias existe entre las acciones de un hacker y las de un cracker si ambos violan los derechos a la privacidad, confidencialidad?

Entre las cuestiones planteadas se encuentra lo referente a la propiedad intelectual (que se relaciona estrechamente con tres conceptos derechos de autor, patentes y marcas) la cual se protegía y protege con el denominado copyright, derecho de patentes y de marca. Qué sucede entonces cuando la información, digitalizada, puede fluir libremente por las redes y burlar estos mecanismo de protección.

Autores como Lévy (2001) plantean que:

"La información, al pertenecer al orden del acontecimiento, situado y dado, en contexto, forzosamente indisociable de una subjetividad, puede, sin duda, cumplir el papel de un servicio remunerado (de formación o de consejo, por ejemplo), pero no de algo de lo que se es dueño, hablando con propiedad. No podemos ser propietarios del momento de un proceso. La disminución de incertidumbre de la teoría de la comunicación es, por naturaleza, absolutamente transitoria y singular. Se podría invocar la propiedad de este texto, no de la información que ustedes saquen de él."

De lo anterior se desprende que se debe tener muy clara la noción de información a manejar a la hora de dictar disposiones legales alrededor de este fenómeno. Basados en el postulado de Buckland podría primero dilucidarse qué es susceptible de protección la información como cosa, proceso y/o conocimiento y de qué manera.

Muchos juristas están abogando por la integración coherente de regulaciones legales y medidas de protección informática como la encriptación de la información, la validación de contraseñas, entre otras. Cabría reflexionar entonces si estas son las soluciones más acertadas.
Siempre queda un resquicio por donde personas de "buena voluntad", aferrados defensores del derecho a la información y otros con no tan buena disposición pueden entrar y acceder a lo restringido. iSe puede considerar a los hacker como personas que incurren en delitos cuando entre sus preceptos está compartir los productos que desarrollen y el conocimiento que poseen? ¿Se debe castigar sólo a los cracker porque no comparten el resultado de su incursión en sistemas de información ajenos y porque destruyen la información valiosa de las organizaciones en las cuales penetran?:Quedan amparadas y justificadas estas acciones bajo el derecho de libertad de expresión y libre acceso a la información?

Las formulaciones y puntos de vista son diversos. El punto neurálgico de la cuestión es el cómo enfrentarlo, cuando la información posee una peculiaridad: El uso no la destruye, y su difusión no hace que quien la tenía la pierda.

Si bien es cierto, como se señala con anterioriad, que el acceso libre y universal a la información es uno de los derechos fundamentales y que la información es un bien público, también es cierto que la información es un bien comercializable, generador de ingresos monetarios. Por tanto, esta dualidad exige que la preservación del acceso y manipulación de la información se practiquen desde una perspectiva ética que refuerza la responsabilidad moral de los individuos y la sociedad.

\section{CONSIDERACIONES FINALES}

La reorganización de la sociedad no implica sólo el perfeccionamiento de la Política y el Derecho como modos de regulación que garanticen y aseguren el supuesto orden del nuevo tipo de sociedad. Implica necesariamente, el perfeccionamiento del individuo, capaz de dominar su propia conducta a través de la regulación moral espontánea, consciente y voluntaria- por la unidad de la esencia humana; por ende, la armonía, el equilibrio y la coherencia social -expresión del progreso moral y social de la humanidad-.

La edificación de esta nueva sociedad requiere de la educación ética y moral del nuevo artífice, al condicionar la formación, aprendizaje e integración de valores de contenido universal que se expresen en cada una de sus acciones -creadoras y transformadoras de la realidad-para la humanización de la sociedad.

Una sociedad que postula la utilización intensiva y extensiva de la información y de las Tecnologías de la Información y la Comunicación. Sin embargo, el uso de estos elementos universalizados es asumido desde y por la singularidad de cada individuo o grupo social; 
por tanto, el control y la orientación del empleo de las mismas -evidentemente regulación moral- ha de lograrse en la medida que los individuos y la sociedad contemplen conscientemente en todas sus acciones el respeto a la integridad y dignidad individual y grupal con un alto sentido de responsabilidad, que implica respeto hacia sí mismo y orienta al redimensionamiento ético en contextos específicos que influyen significativamente, en las relaciones sociales dentro del marco de la Sociedad de la Información.

Congruentemente, la humanidad tiene ante sí el reto estratégico de solucionar los problemas que provoca la división derivada de la posibilidad y capacidad para acceder y utilizar la información; a nivel táctico debe implementar mecanismos que minimicen las probabilidades de profundización de estos problemas, que reduzcan la brecha informacional.

Una de las conclusiones arribadas por los miembros del foro de Consulta sobre Derecho e Informática (1996) es que: "si bien es importante legislar, más importante aún puede ser la culturización de la sociedad. Por ello la legislación debe de ir acompañada de campañas y programas de cultura informática, con la finalidad de minimizar el delito informático, así como mejorar el uso y adquisición de las tecnologías de la información".

A ese planteamiento sería interesante modificar ciertos elementos:

- no lastrar la culturización de la sociedad solo con la cultura informática, acertado es promover la cultura informacional, y por tanto pensar en

- no sólo mejorar el uso y adquisición de las tecnologías de la información, sino de la información en sí más allá de soportes, tecnologías para su tratamiento y canales de transmisión. Adaptar y concienciar al usuario que al enfrentar el mundo de la información debe ejercitar los valores éticos establecidos, cumplir con las disposiciones legales establecidas para ese contexto y respetar los derechos inalienables de todo ser humano referentes a la información.

No obstante, a esta construcción de la cultura informacional se le presentan barreras tecnológicas, económicas y materiales (infoestructura y presupuesto), barreras culturales y gnoseológicas (analfabetismo informacional).

Así pues, la alfabetización informacional como antesala de la cultura informacional aparece como el proceso que permite responder a los desafíos éticos, políticos y legales contemporáneos: la infodivisión y el capitalismo informacional, la propiedad intelectual y la economía de información, la libertad de expresión, los delitos informáticos y las tradiciones morales, el acceso universal y el gobierno de la red.

La alfabetización informacional promueve la democratización social, al proveerle a los ciudadanos aquellos componentes cognoscitivos y culturales (incluido los éticos) que le da la opción de elegir su nivel de participación societal. La exclusión permeada por las situaciones de acceso a la información quedará solo en la capacidad que tengan la sociedad y sus organizaciones sociales de gestionar las tecnologías y sus sustitutos.

En esta concepción de la realidad influye significativamente, la educación moral, al contribuir a la formación de valores de contenido universal que "regulen y orienten la actitud de los individuos hacia la reafirmación del progreso moral y el crecimiento del humanismo" (Chacón, 1999) -síntesis sinérgica de valores como la solidaridad, la honestidad, la responsabilidad y la justicia, en la real identidad individuogénero-, que conllevan al perfeccionamiento humano que ha de tributar, sin duda alguna, al perfeccionamiento y progreso de la sociedad.

Artigo recebido em 23/11/2004 e aceito para publicação em 15/08/2005. 


\section{Yohannis Marti / Rosa Lidia Vega-Almeida}

\section{REFERÊNCIAS}

BIOMUNDI. La industria de la información: el mundo en cifras y hechos. La Habana: IDICT, 1999.

CAPURRO, R. Contribuciones a una ética de la información. 2002. Disponível em: < http://www.capurro.de/eticainf.ppt>.

Acesso em: 3 set. 2003.

CHACÓN, N. Formación de valores morales. La Habana: Editorial Academia, 1999. p.1-53.

ERICHSEN, M; REZENDE, A. M.; BÓREM, G. A. Estudos cognitivos en ciência da informação. Encontros Bibli, n. 15, 2003. Disponível em: <http:// www.encontros-bibli.ufsc.br/Edicao_15/borges_estudoscognitivos.pdf $>$. Acesso em: 20 jun. 2003.

GONZÁLEZ, D. La calidad en la educación. Lima: GRAFIMAG, 2000.

GONZÁLEZ, M.; LÓPEZ, J.; LUJÁN, J. Ciencia, tecnología y sociedad: una introducción al estudio social de la ciencia y la tecnología. Madrid: Editorial Tecnos, 1996.

LÉVY, P. El anillo de oro: inteligencia colectiva y propiedad intelectual. 2001. Disponível em

$<$ http://www.sindominio.net/biblioweb/telematica/levy.html $>$.

Acesso em: 3 set. 2003.
LINARES, R.; PATTTERSON, M.; VICIEDO, T. La información através del tiempo. La Habana, v. 3, n. 5, 2000.

MEMORIAS del foro de consulta sobre derecho e informática. In: FORO de consulta sobre derecho e informática, México, octubre 1996. Disponível em: <http://www.cddhcu.gob.mx/camdip/foro/df/df_pcs.htm>. Acesso em: 2 ago. 2003.

MERRILL, J. C. El periodista apolonisiaco. In: MERRILL, J.; BARNEY, R. La prensa y la ética. Buenos Aires: Editorial Universitaria, 1981.

MOREIRO, J. A. Introducción al estudio de la información y la documentación. Antioquia: Universidad de Antoquia,1998.

MORIN, E. Los siete saberes necesarios para la educación del futuro. Paris: UNESCO, 1999.

NAÇÕES UNIDAS. Proyecto de declaración de principios. Disponível em: $<$ http://www.itu.int/dms_pub/itu-s/md/03/wsispc3/doc/S03-WSISPC3DOC-0002!!MSW-S.doc $>$.

Acesso em: 2 ago. 2003.

. Conselho Econômico e Social. Development and international cooperation in the twenty-first century: the role of information technology in the context of a knowledge-based global economy: report of the SecretaryGeneral United Nations. New York, 2000.

RUBESTEIN, R. H. La psicología: métodos, principios y técnicas. Moscú: Academia, 1964. 